\title{
TSO-DSO coordination to acquire services from distribution grids: simulations, cost benefit analysis and regulatory conclusions from the SmartNet project
}

\author{
Marco Rossi*, Gianluigi Migliavacca*,Giacomo Viganò*, Dario Siface*, \\ Carlos Madina , Inés Gomez , Ivana Kockar", Andrei Morch \\ * Ricerca sul Sistema Energetico (RSE S.p.A.), Milan, Italy \\ †TECNALTA, Derio, Spain \\ 『University of Strathclyde, Glasgow, United Kingdom \\ §SINTEF Energy Research, Trondheim, Norway
}

\begin{abstract}
The continuously growing distributed generation and the business potential for demand response are gradually enabling significant provision of flexibility and reserve towards distribution networks. For this reason, transmission and distribution system operators need to coordinate their operation in order to develop efficient market arrangements that can help utilize all the resources capable of providing ancillary services. SmartNet project investigated the potential interaction schemes between network operators, together with the possible new services devoted to the optimal distribution grid management. This paper summarizes the main challenges in simulating complex electricity systems and flexibility markets for three European countries (Italy, Denmark and Spain) in 2030 scenarios. The simulation results are then analyzed using cost-benefit analysis and regulatory conclusions are deduced.
\end{abstract}

Index Terms-Aggregation of distribution resources; ancillary services market; network simulation; network operators coordination schemes

\section{INTRODUCTION}

The steadily increasing renewable generation share is modifying the way of planning and operating the electricity systems. This shift has brought on a significant level of generation being provided by stochastic renewable distribution generation, increasing expected levels of the reserve to be procured at distribution level. This evolution is also driven by the business potential for demand side management and the envisaged possibility for distribution operators to activate medium/low voltage flexible resources in order to solve local congestions [1]-[5]. In order to manage the services requested for all the system voltage levels, Transmission and Distribution System Operators (TSOs and DSOs respectively) need to coordinate their actions by means of optimized and formal architectures which foresee exchange of data, provision and prioritization of services, shared market schemes, etc. A number of possible TSO-DSO Coordination Schemes (CSs) have been investigated [4]-[6] with general conclusions on their implementations drawn in [2] and practical experience of power sector stakeholders [5],[7]. In particular, it is demonstrated that TSO-DSO coordination is always beneficial [1],[2],[5],[6],[8], with the potential negative impacts of uncoordinated actions in terms of system operation and commercial market player business discussed in [3].

The paper focuses on the experience and outcomes of the European H2020 project SmartNet (http://smartnetproject.eu/), which has been one of the pioneers in the investigation of TSO-DSO coordination. In particular, the project dealt with balancing and congestion management services (for both transmission and distribution systems) which have been addressed simultaneously by optimizing the power reserve needed by both TSOs and DSOs. The choice of a single flexibility market for multiple services guarantees an optimized activation of resources (in terms of volume/costs) with respect to a separated management of the services [7]

\section{A. TSO-DSO coordination schemes}

There are a number of potential TSO-DSO coordination schemes that can be used to procure and activate different resources at distribution level for ancillary services. The SmartNet project selected and evaluated four CSs [9], which are summarized as follows:

CS A Centralized ancillary services market model

The DSO is not involved in procurement of flexibility, with distribution market products only being purchased by the TSO for balancing and congestion management at transmission level.

CS B Local ancillary services market model

Two-step procedure, where DSO has priority to buy flexibility for the management of distribution grid services (congestion management). The remaining flexibility (including the un-activated distribution one) can be procured by the TSO through a separate market aimed at activating balancing and congestion management services for the transmission network. 
CS C Shared balancing responsibility model

DSO and TSO manage their system separately, both performing balancing and congestion management. The distribution balancing service is operated by scheduling the power exchange between transmission and distribution network (power profile that is agreed between the two network operators).

CS D Common TSO-DSO ancillary service market model DSO and TSO access the same market to buy flexibility aimed at solving congestions and balancing. As CS B, the DSO is responsible for the congestion management only at distribution level, and the TSO uses the same market to procure services it needs.

As outlined above, the main differences among the proposed CSs consist of the roles, involvement and priorities of the distribution/transmission network operators. This has an impact on the way the market is organized, and on the type of the provided services, e.g. congestion and balancing management specifically devoted to distribution network operation. Indeed, DSOs are the actors for which the behavior is expected to change significantly with respect to the adopted $\mathrm{CS}$, and a detailed analysis on the DSO involvement and implications is discussed in [5] and [9].

\section{B. 2030 Energy/flexibility scenarios}

The effectiveness of the investigated CSs highly depends on the scenario in which they are applied. In particular, the condition of distribution network is expected to play a fundamental role in defining the most promising TSO-DSO interaction. In order to investigate the dependency of scenarios on the CSs performance, SmartNet project considered three reference countries (Italy, Denmark and Spain) with their foreseen 2030 electrical evolution [10]. With respect to the current situation, the main assumed changes are:

- Solar and wind generation increase (mostly located at distribution level), which will be comparable to the total capacity of conventional power plants in Italy and Spain.

- Increase of storage-based technology, especially due to the roll-out of electric vehicles.

- Management of flexible thermal loads, particularly significant in Denmark where large cogeneration units are expected to be replaced by controllable heat-pumps.

\begin{tabular}{|c|c|c|c|}
\hline & Italv & Der & no \\
\hline $\begin{array}{c}\text { Transmission } \\
\text { network }\end{array}$ & $\begin{array}{l}150 / 220 / 380 \mathrm{kV} \\
3648 \text { buses } \\
4230 \text { branches } \\
115 \mathrm{GW} \mathrm{mFRR}\end{array}$ & $\begin{array}{l}150 / 220 / 380 \mathrm{kV} \\
144 \text { buses } \\
198 \text { branches } \\
11 \mathrm{GW} \mathrm{mFRR}\end{array}$ & $\begin{array}{l}150 / 220 / 380 \mathrm{kV} \\
1537 \text { buses } \\
2231 \text { branches } \\
74 \mathrm{GW} \mathrm{mFRR}\end{array}$ \\
\hline $\begin{array}{c}\text { Distribution } \\
\text { network }\end{array}$ & $\begin{array}{l}15 / 20 \mathrm{kV} \\
6245 \text { buses } \\
\text { and branches } \\
14 \mathrm{GW} \text { mFRR }\end{array}$ & $\begin{array}{l}10 \mathrm{kV} \\
3046 \text { buses } \\
\text { and branches } \\
8 \mathrm{GW} \mathrm{mFRR}\end{array}$ & $\begin{array}{l}15 / 20 \mathrm{kV} \\
2799 \text { buses } \\
\text { and branches } \\
10 \mathrm{GW} \mathrm{mFRR}\end{array}$ \\
\hline
\end{tabular}

The details on how this technology evolution is distributed over the three considered territories and, consequently, on the electricity networks are presented in [10] and summarized in
Table I. Analysis of the main characteristics of the current national distribution network systems and the available balancing/congestion management reserve show that:

- In Italy, large volumes of flexible technology are expected to be located at distribution level. This flexibility, in addition to transmission system services, has the potential to support congestion management on distribution networks at a competitive price.

- In Denmark, the flexibility located at distribution level is significant (if compared to the one available at high voltage level). Most of it is represented by electric vehicles and thermal loads which have lower flexibility margins with respect to other device typologies (e.g. renewables) since charging and temperature constraints jeopardize the amount of flexible power that can be provided at given time instant.

- Spain will have a significant amount of flexibility located on the distribution network. A medium-voltage network structure and flexibility portfolio similar to the Italian one is assumed, but the lower distribution flexibility (compared to Italy) determines small congestion management reserves for these voltage levels.

Table I also provides the topological characteristics of the simulated systems. In order to optimize the analysis of the scenario and the simulation burden, distribution networks of areas without congestions have been modelled as single-node aggregations of devices (i.e. copper-plate assumption). The remaining portions of medium-voltage distribution system have been represented in detail, while low-voltage devices have been aggregated together, corresponding to the related secondary substations.

The particularities of these three investigated power systems are expected to have an impact on the feasibility of each TSO-DSO coordination scheme. For this reason, once the 2030 scenarios have been developed, dedicated simulations have been carried out in order to define, for each country, the most promising interaction between network operators. The next sections outlines the main aspects related to the simulation activity (Section II) and cost-benefit analysis (Section III), whose results supported the regulatory investigation summarized in Section IV.

\section{SIMULATION OF TSO-DSO COORDINATION SCHEMES}

Because of the need to test performances of TSO-DSO interactions, a simulation platform capable of considering both transmission and distribution network components has to be used. In addition to the grid elements, the hundreds of thousands of distribution devices (potentially contributing to ancillary services) have also to be modeled, considering both their internal dynamics and interaction with the market. Thus, a dedicated three-layer simulator structure, capable of modeling various market dynamics, has been developed, as shown in Figure 1, and discussed in Section II.B [11].

As outlined above, the simulations for a number of scenarios have been carried out in order to evaluate the performance of TSO-DSO coordination schemes in activating flexibility for the management of balancing and congestion 
issues. Both automatic and manual Frequency Restoration Reserves (aFRR and mFRR, respectively) have been optimized to provide the required services.

Another dimension that plays an important role in the actuation of the coordination schemes is represented by the communication infrastructure. For the considered services, the present technology has been proven to be adequate for the activation of the requested reserves [12] and, as reported in [13], no significant differences are expected in communication requirements for all the investigated CSs. For this reason, simulations have not directly considered communication layer.

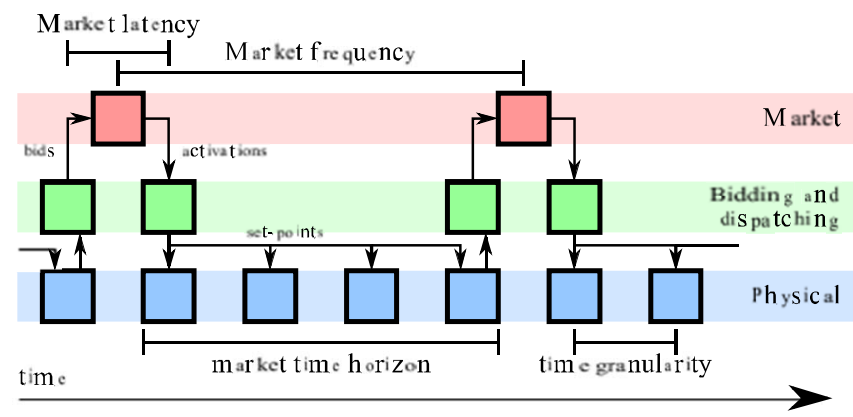

Figure 1. Three-layers structure of the proposed simulator

\section{A. Bidding and dispatching layer}

The interface between the physical power units and the market is represented by the bidding and dispatching algorithms which, especially for the distribution devices, implement aggregation and disaggregation routines. Having considered the limited/specific degree of flexibility of some technologies, aggregation strategies can significantly increase the competitiveness of resources that, if acting alone, would not be exploitable for ancillary services. This is the case of power units for which a deviation with respect to the baseline profile involves significant rebound effects that can be minimized by means of an optimal management of other flexibilities within the same aggregation portfolio. The main aspects related to the bidding strategies are shown in Table II.

\section{B. Market layer}

The role of this layer consists of running the clearing routines aimed at selecting the optimal activations of submitted bids (representing the available mFRR) for the solution of the imbalance and congestions predicted for the next time steps. Normally, this market also manages aFRR bids to guarantee the operation of secondary frequency control without causing congestions. However, in order to simplify the simulation process, a separate off-line simulation of aFRR procurement is carried out (see section III.B).

The way of clearing the market is dependent on the adopted TSO-DSO coordination scheme (see Table III), however, the main steps followed by this layer procedures can be summarized in the following generic way:

1. The network status is calculated for the time steps of the market horizon on the basis of the most current forecasting (forecasting error is simulated according to real statistics and discount factors [10]).
2. Optimal power flow is run in order to solve the predicted imbalance and congestions by using the available mFRR. For these, Mixed Integer Linear Programming models are adopted [11], i.e. DC optimal power flow for meshed networks (transmission) and linear approximation of AC optimal power flow for radial ones (because of the necessity of considering voltage congestions at distribution level [8]).

3. Acceptance of bids is communicated back to the bidding and dispatching layer. When network asset is available (static compensators, tap-changing transformers, etc.) the market layer takes them into account and their resulting set-points are sent directly to the physical layer.

Because of the adoption of a mixed-integer solver, complex bids (which include constraints such as time deferability, rebound effects, etc.) can be easily processed taking into account the consequences of their activation (which can be significant for some flexible-demand technologies [14]). Finally, as shown in Figure 1, the impact of market timing dynamics can be explored in terms of:

- Market latency - time taken by the clearing algorithm to process bids and define the optimal activations.

- Market frequency - time indicating how often bids are collected and clearing routines are launched.

- Market horizon - time interval for which the optimal solution is calculated (horizons larger than one time step guarantee the management of rebound effects, time deferability and integral constraints).

\section{Physical layer}

Once market activations are translated to set-points for the flexible resources, the physical layer updates their internal state and processes the consequent network evolution. The first algorithms executed by this layer consists of calculating the power output of each device connected to the network:

- Flexible resources receiving power set-points from the dispatching routines

In this case the power set-point is compared with the actual capability of the device and corrected if needed. The internal state of the power unit (e.g. the accumulated energy of a storage-based device) is updated accordingly.

- Flexible resources receiving non-power set-points (e.g. temperature target) from dispatching routines

In this case the model of the device is used in order to calculate the correspondent power value, which is eventually compared with the electrical capability of the simulated actuator. If power correction is needed, the internal state of the device (e.g. thermal variables) are updated accordingly by reversing the model.

- Non-flexible resources

For resources that are not participating to the ancillary services market, a zero-state fixed-power model is used.

For all the simulated resources, a time evolving forecasting error is applied. The working principle of the error model is based on the addition of random values (which magnitude is evaluated on the basis of real observations [10]) on the actual profiles of devices quantities. 
TABLE II. SIMULATED FLEXIBLE POWER TECHNOLOGY

\begin{tabular}{|c|c|}
\hline Tecl & Description of flexibility and aggregation process \\
\hline $\begin{array}{l}\text { Conv } \\
\text { gener }\end{array}$ & $\begin{array}{l}\text { A traditional bidding and dispatching routine is } \\
\text { implemented for interfacing conventional generators with } \\
\text { the market. Cost of fuel and carbon emission penalties } \\
\text { are considered, together with the maximum/minimum } \\
\text { power and ramp constraints [15]. }\end{array}$ \\
\hline Ator & $\begin{array}{l}\text { Atomic loads are characterized by a fixed consumption } \\
\text { profile, which cannot be changed but can be deferred in } \\
\text { time. Thanks to optimal combination of the flexibility of } \\
\text { several atomic loads, the resulting rebound effect } \\
\text { (summation of the rebound effects of all the controlled } \\
\text { loads) can be minimized/canceled and aggregators can } \\
\text { potentially propose conventional quantity/price bids [16]. }\end{array}$ \\
\hline $\begin{array}{l}\text { Com } \\
\text { and } 1\end{array}$ & $\begin{array}{l}\text { A bidding approach, similar to the one adopted for } \\
\text { conventional generators, is adopted here. In this case. } \\
\text { additional constraints, mostly related to the requested } \\
\text { thermal demand, are considered. Rebound effects } \\
\text { information are included within the submitted bids in } \\
\text { order to allow their consideration within the market } \\
\text { clearing routines [15]. }\end{array}$ \\
\hline loads & $\begin{array}{l}\text { All the loads aimed at controlling the temperature of a } \\
\text { building/device/fluid are aggregated by a bidding routine } \\
\text { which includes their thermal model (monitoring is } \\
\text { required). The bids are generated on the basis of the } \\
\text { current working point of each controlled units and on the } \\
\text { possible discomfort/rebound effect deriving from a } \\
\text { baseline deviation [17]. }\end{array}$ \\
\hline $\begin{array}{l}\text { Electricity } \\
\text { storage-based } \\
\text { units }\end{array}$ & $\begin{array}{l}\text { A single algorithm is dedicated to the aggregation of } \\
\text { power units capable of storing electricity, independently } \\
\text { on its final use. Hydro pumped generation, static storage } \\
\text { devices, electric vehicles, etc. are managed by means of } \\
\text { the same optimization problem and on the basis of the } \\
\text { actual availability/constraints of the controlled units (i.e. } \\
\text { electric vehicles are not always connected to the grid and } \\
\text { have charging time constraints) [18]. }\end{array}$ \\
\hline $\begin{array}{l}\text { Curtailable } \\
\text { generators and } \\
\text { loads }\end{array}$ & $\begin{array}{l}\text { Solar, wind, small-scale hydro and some load typologies } \\
\text { (e.g. dimmable lights) can be curtailed without rebound } \\
\text { effects. In this case, a conventional bidding strategy can } \\
\text { be adopted, without the necessity of specifying logical } \\
\text { constraints and rebound information [19]. }\end{array}$ \\
\hline
\end{tabular}

TABLE III. MARKET PROCESSING CHARACTERISTICS FOR DIFFERENT TSO-DSO COORDINATION SCHEMES

\begin{tabular}{|l|l|}
\hline CS A & $\begin{array}{l}\text { TSO-DSO COORDINATION SCHEMES } \\
\text { Bids are collected by aggregating resources with the granularity } \\
\text { of transmission network nodes. Only system imbalance and } \\
\text { transmission network congestions are solved by the market } \\
\text { clearing routine. }\end{array}$ \\
\hline CS B & $\begin{array}{l}\text { Bids are collected by aggregating resources with the granularity } \\
\text { of distribution network nodes. First, distribution services } \\
\text { (congestion management) are processed by local markets, while } \\
\text { the non-selected bids are aggregated and forwarded to the } \\
\text { central market aimed at solving system imbalance and } \\
\text { transmission network congestions. }\end{array}$ \\
\hline CS C & $\begin{array}{l}\text { Distribution and transmission networks are managed separately } \\
\text { as independent systems. Bids collected at low/medium voltage } \\
\text { levels are processed in order to solve only distribution network } \\
\text { imbalance and congestions. Bids collected at high voltage levels } \\
\text { are processed to solve only transmission network imbalance and } \\
\text { congestions. }\end{array}$ \\
\hline CS D & $\begin{array}{l}\text { Bids are collected by aggregating resources with the granularity } \\
\text { of distribution network nodes. Both distribution and } \\
\text { transmission services are processed simultaneously by the same } \\
\text { market clearing routine. }\end{array}$ \\
\hline
\end{tabular}

Once the power exchange of each connected (flexible and non-flexible) unit is available, the network status can be computed by running a simple power flow simulation. In theory, the power exchange of some resources can be dependent on the network status and more advanced simulation techniques might be required [20]. However, it is assumed that all devices participating to system services can communicate with network operators SCADAs, and local controllers (e.g. volt-var/volt-watt controllers) are not activated in normal working conditions.

Having considered that the power exchange of each device is subject to forecasting error, unpredicted loading/voltage congestions can occur, and operators are called to solve them promptly. The possible measures consist of activating network asset (which normally does not impact on the services activated by the market) and, if not enough, re-dispatching flexible resources (with an impact on the activations requested by the market). According to Figure 2, the simulation of these measures can be performed by running an optimal power flow (instead of a simple power flow) which objective function can be constructed to guarantee the following action priority:

1. Tap-changing and phase-shifter transformers, mostly for the management of voltage issues at distribution level and loading congestions on transmission grid respectively.

2. Reactive power of static compensator and flexible resources, for voltage control at any voltage level (reactive power is assumed to be a non-remunerated reserve).

3. Active power control (re-dispatching) of flexible units, for the management of overloading issues.

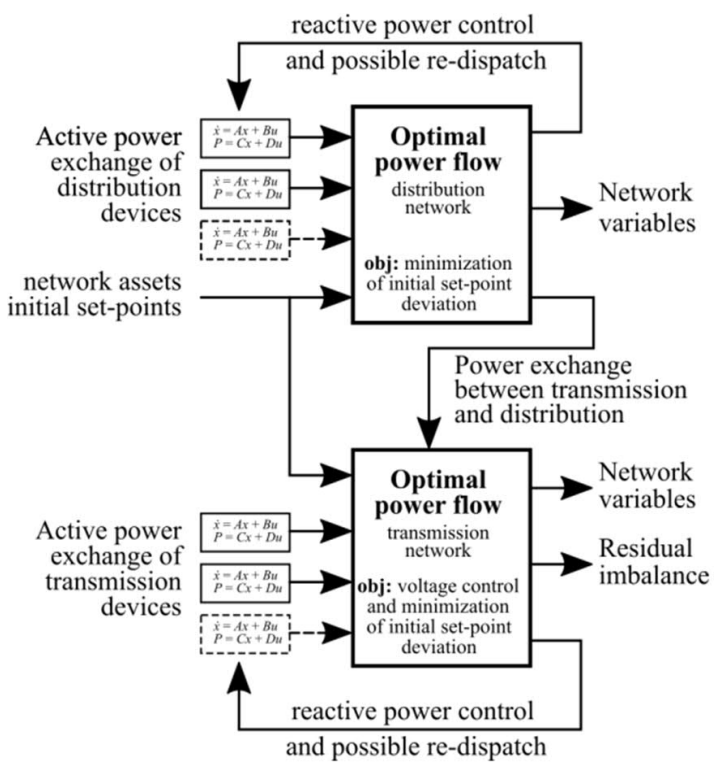

Figure 2. Block diagram of the network physics simulator

Based on the strategy summarized in Figure 2, the simulator returns the network status including also the possible actions (active/reactive power re-dispatching) taken by network operators in case of unforeseen congestions. In 
particular, having considered the free-of-cost reactive power flexibility, its optimal management allows the solution of a significant portion of distribution network problems (especially voltage issues) even in CSs with marginal DSO market involvement.

\section{Simulation Results And Cost Benefit AnAlysis}

Because of the complexity of the selected scenario (which includes both transmission and distribution networks, several thousands of controllable resources including the physics behind them) the resulting simulation became computationally expensive. For this reason, the complete scenario has been reduced in order to find a compromise between simulation time and representativeness. According to the analysis reported in [10], the quantity subject to the highest variability is represented by the power profile of renewable energy sources and 3 typical days (subject to different occurrences within the year) have been considered representative of a one-year scenario. In addition to this measure, static resources (the ones with no internal states) have been lumped if connected to the same network node.

Once the scenario datasets have been adjusted, the market dynamics have been defined. The simulation has been carried out for the three selected countries with a time granularity of 15 minutes, having considered:

- A market frequency and horizon of 60 minutes (the market clearing is performed every hour and it optimizes the activations of each 15 -minutes slot of it).

- A latency of 15 minutes (bids are submitted 15 minutes before the market clearing process).

As discussed above, the market acts on the foreseen imbalance which has been predicted on the time instant in which the clearing algorithm is launched. According to the adopted model of forecasting error, it differs from the actual one as depicted in Figure 3.

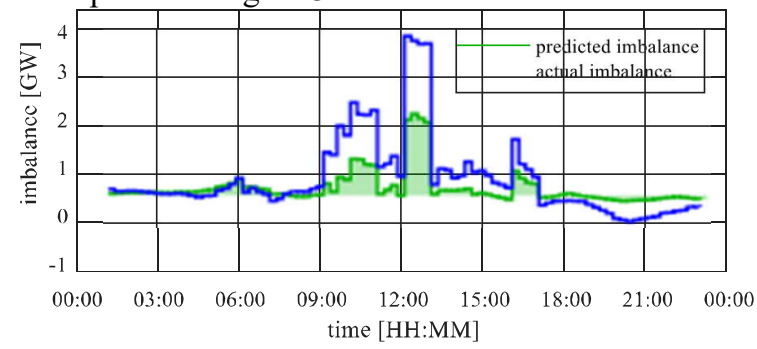

Figure 3. Imbalance predicted (and solved) by the market, compared with the actual mismatch between generation and load

\section{A. Market activations ( $m F R R)$}

In order to balance the network, the market layer processes the available upward/downward mFRR depending on the imbalance sign. However, the presence of congestions determines situations in which mFRR is activated in both the directions simultaneously. This is particularly evident in Figure 4 where, because of transmission network congestions, large bidirectional volumes of $\mathrm{mFRR}$ are activated in the early morning and evening even if the expected imbalance is low.
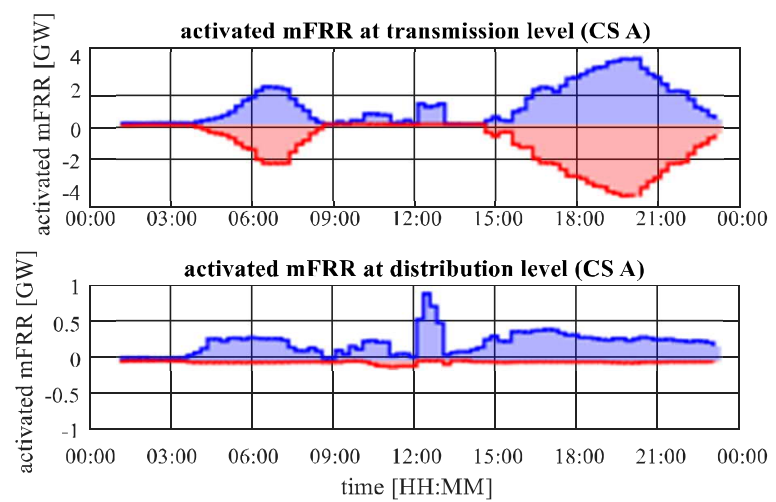

Figure 4. Activated $\mathrm{mFRR}$ at transmission and distribution levels
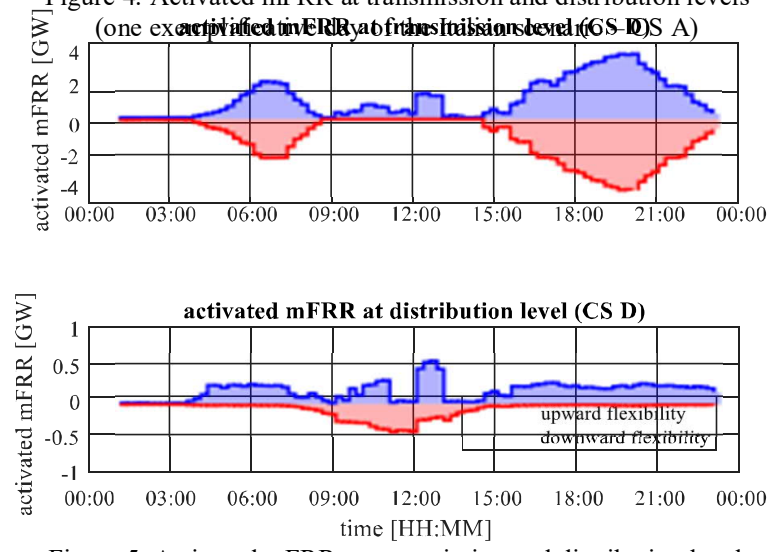

Figure 5. Activated $\mathrm{mFRR}$ at transmission and distribution levels (one exemplificative day of the Italian scenario - CS D)

According to the simulation outcomes, upward mFRR located at distribution level proves to be competitive with respect to the one located on the transmission network. In fact, when no congestions occur, similar volumes of upward distribution/transmission mFRR are activated. However, the presence of distribution constraints limits the availability of low/medium voltage resources. This effect can be noticed by comparing the results of CS A (Figure 4) with the ones of CS D (Figure 5). In fact, the introduction of congestion management services on distribution networks determines lower upward activations for these voltage levels. On the contrary, a significant increase can be noticed for distribution downward mFRR and unequivocally attributed to grid congestions due to solar generation. As expected, by increasing the amount of services, mFRR optimal activations rise in cost. Having adopted a pay-as-clear remuneration scheme based on nodal prices [11], it can be noticed that costs increase with the market complexity. Figure 6 reports the results of the full-year Danish case, where:

- CS A is the cheapest one, since it deals with transmission services only.

- CS B and CS D manage also distribution congestion management, resulting in higher costs (CS B splits the services in two separated markets, determining sub-optimal solutions and slightly higher costs).

- $\mathrm{CSC}$ is managed as CS B, having also included balancing services for each single distribution network. 


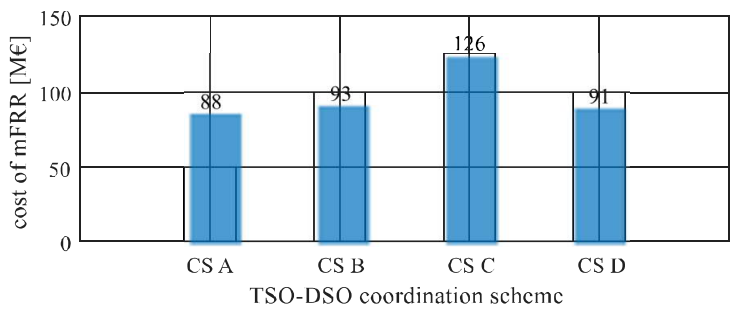

Figure 6. Cost of activated mFRR (Danish case)

\section{B. Cost of residual imbalance (aFRR)}

The additional costs of $\mathrm{mFRR}$ activations can be attributed to benefits in terms of system management. In fact, by adding services to the optimization problem solved by the market, less issues remain to be solved by separated (and less optimal) processes. This is the case of congestion management at distribution level which, if not optimized together with the other services processed by the market, determines manual actions by network operators. Specifically, the active power re-dispatch of resources inevitably results in residual imbalance to be added to the one due to forecasting error. For this analysis, the reducing system imbalance is considered the major benefit.

Residual imbalance is normally managed by means of aFRR, which is activated by automatic controllers but procured through the same market dealing with mFRR. The integration of aFRR within the simulator described above would significantly increase the complexity of the clearing routines, with a consequent impact on the simulation time. For this reason, a simplified off-line procedure for the calculation of aFRR volumes and costs have been defined [21] (Figure 7):

- Activated aFRR is assumed to be equal to residual imbalance. For each hour (market frequency) the total available aFRR volume is conservatively considered to be twice the activated one.

- aFRR flexibility bids are calculated in proportion to submitted mFRR bids. According to experience [21], costs and volumes of these two reserves can be considered proportional.

- The cheapest aFRR bids are selected in order to cover the reserve needs (off-line aFRR market simulation).

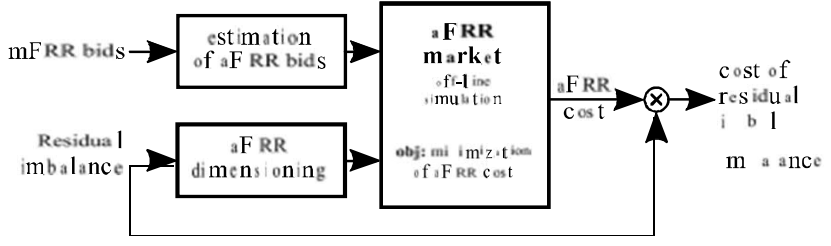

Figure 7. Block diagram of the algorithm used for the calculation of residual imbalance cost

Because of this approach, the residual imbalance can be monetized and added to the other cost figures considered by this analysis. Figure 8 reports exemplificative results, which show how CS B and CS D allow lower aFRR activations and costs with respect to CS A (where distribution resources re-dispatch makes mFRR balancing less effective).

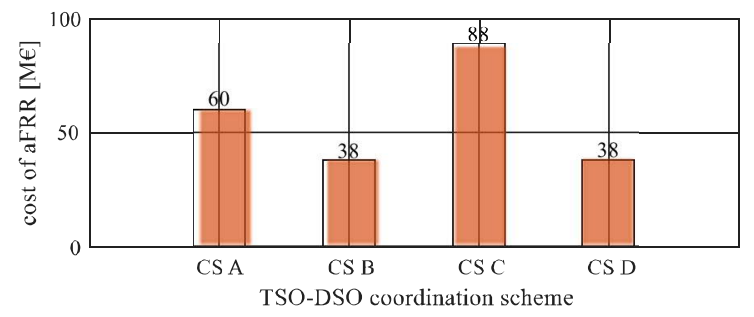

Figure 8. Cost of activated aFRR (Spanish case)

According to the simulation results obtained for the three countries, CS C typically returns high costs for both mFRR and aFRR activations. This phenomenon can be explained by observing that the simulated market is unable to balance the considered system (Figure 9) because of the limited available resources (DSO can use distribution flexibility only, TSO can use transmission flexibility only).

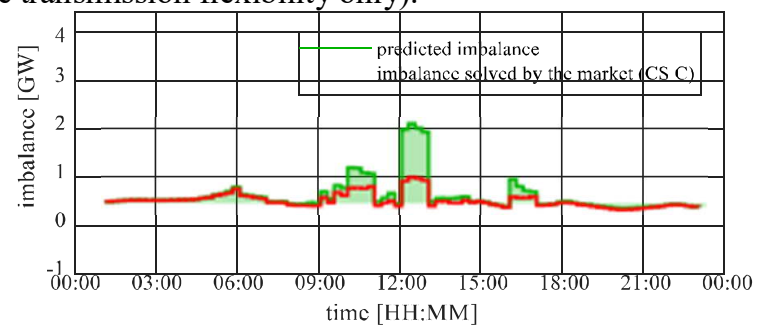

Figure 9. Predicted imbalance compared with the activations processed by CS C markets

\section{Cost of information technology}

In order to allow the participation of distribution resources to ancillary services markets, current Information Technology (IT) has to be adapted in order to deal with aggregation processes and complex market clearing routines. One possible IT cost calculation approach consists of the Constructive Cost Model, which application on the investigated CSs is reported in [13] together with the calculation of the related annuity cost. As anticipated above concerning communication infrastructure, all the CSs are assumed to be similar in terms of requirements and costs and they have not been object of investigation within the proposed cost-benefit analysis.

\section{Total costs $(m F R R+a F R R+I T)$}

Thanks to the simulator presented within the previous section, the impact of the considered CSs on the three reference countries have been investigated and the resulting cost figures have been reported in Figure 10.

In Italy, the highest performance of CS B and CS D is evident, and this is happening due to the large amount of distribution flexibility that is constantly procured for local congestion management. It is also noticeable how an optimized management of distribution resources significantly decreases the amount of residual imbalance (aFRR activations) with respect to $\mathrm{CS} A$.

Denmark, instead, shows a counterintuitive behavior. In fact, TSO-DSO coordination schemes that are supposed to be more optimal than CS A, demonstrates a lower performance (even excluding IT costs). According to the scenario (1) 
assumptions, most of the distribution reserve is represented by electric vehicles and thermal loads, which have limited flexibility if compared to photovoltaics (which is the highest distribution reserve in the other two countries). For this reason, the distribution network is assumed to be robust, with a congestion probability lower than the forecasting error on balancing. This means that there is a concrete risk that the market overestimates the limitations of the distribution systems, determining a slightly increase of mFRR cost.

Finally, Spain represents a middle ground between the Italian and Danish scenarios. In this case, the adoption of market architectures which include distribution constraints has its advantages. Nevertheless, these benefits are cancelled by the higher IT complexity and costs. This phenomenon is not making evident the added value (in terms of costs) of adopting a complex CS rather than the simplest CS A. This conclusion is applicable to all the situations in which reserves activations lead to similar costs among coordination schemes. In this case, the adoption of complex CS is however beneficial since it provides additional observability/controllability of the system.
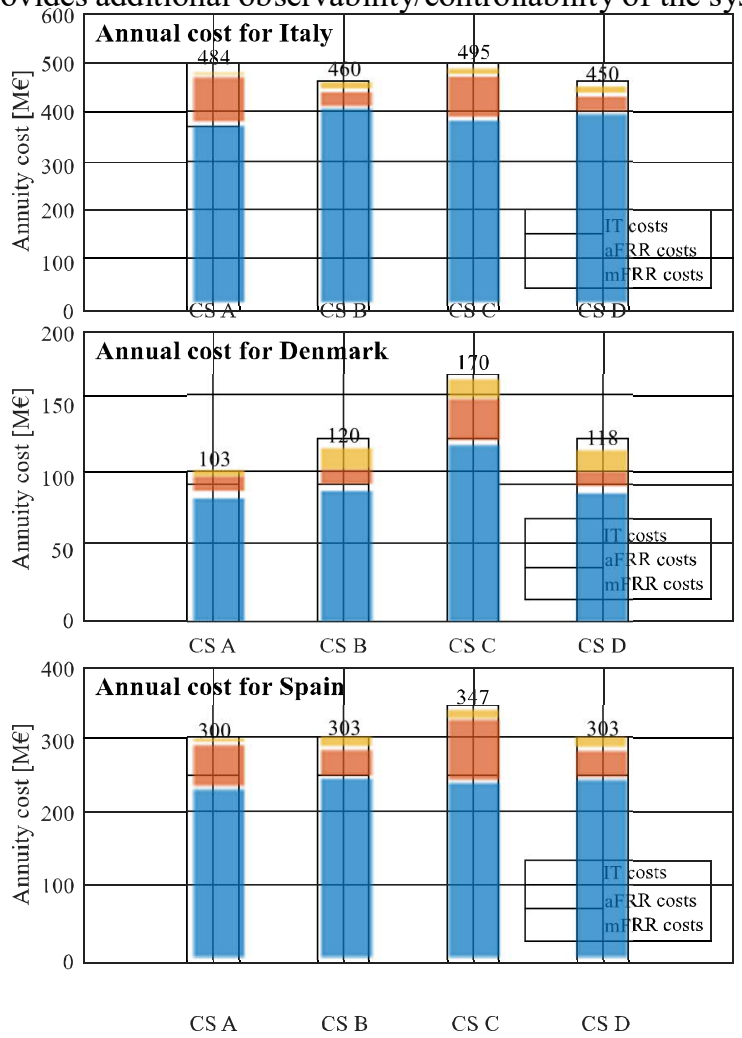

Figure 10. Annual total costs for balancing and congestion management in Italy, Denmark and Spain (2030 scenario)

In all these scenarios, CS C is definitively the coordination scheme with the lowest performance. As anticipated above, distribution reserve is not often capable of guaranteeing an effective balancing service. This determines high residual imbalance (resulting in high aFRR costs) and, as for the Danish case, the activation of high-cost mFRR bids when local services experience scarcity of resources.

\section{REGULATORY CONCLUSIONS}

Following the results shown in the previous section, a list of remarks mainly, but not only, related to regulation has been drawn and summarized in the following paragraphs.

\section{A. From CAPEX to CAPEX+OPEX}

The simulation results (in particular the Italian case) showed clearly that the use of flexibility resources is able to provide DSO more options to solve network issues, than the simple network refurbishment. Even in CS A, where DSO is not (or only partially) involved in ancillary services market, the distribution operator must have a full control over its network, and for this purpose appropriate investments in IT are needed. It would be important to enable a DSO to compare such investments with the ones for the network expansion, considering a long-term planning perspective. This also needs to be carried out in coordination with the transmission level so to capture efficiency of the whole system. Regulation should thus consider the experience of SmartNet, which provides an additional proof that combined operation-capital expenditures (OPEX and CAPEX respectively) can be competitive with respect to the conventional only-CAPEX approach.

\section{B. Local markets illiquidity}

All the considered scenarios may be affected by scarcity and illiquidity of resources at distribution level, especially for the provision of local balancing services. In particular, this problem is relevant for those CSs that implement any degree of separation between local and global markets, that is CS B and CS C. The solution is to increase as much as possible the volume and number of resources participating in the local markets. This aim could be achieved by, for example, enlarging the perimeter of the market by involving small DSOs to join up in a single, and wider, local market; and/or by introducing products tailored on the technical needs of the less flexible resources, particularly looking at the demand side.

\section{Separation between global and local markets}

The separation between global and local markets also bring forth sub-optimality problems. In CS B, after DSOs activate the needed resources to solve local congestions, the remaining ones are made available to TSOs for the global balancing market, that takes place in a second step. This two-step procedure reduces the economic efficiency of the system (as also demonstrated in [8]). In fact, CS B makes it impossible to take advantage of the local congestion management to simultaneously solve a possible imbalance somewhere else in the system, and reduces the amount of resources activated globally (e.g. as is the case in CS D). Furthermore, local and global markets may also have different timing. In this case, they have to be adequately coordinated in order to avoid double acceptance of bids presented on both markets (e.g. as observed in [22] and [23]).

The problem is even more prominent in CS C, which is set up with two separated markets for transmission and distribution services, with a fixed exchange profile imposed at the boundary between the two. This hinders compensation of 
local and global imbalances with the opposing signs. The firm constraint imposed at the boundary between transmission and distribution is very strong and may result in a significant loss of optimality (and even rendering a problem infeasible). Adding degrees of flexibility at this boundary could be beneficial for the efficiency of such a scheme, but it is difficult to preserve the complete separation between the two markets. Nevertheless, having assumed a remuneration scheme based on nodal prices, this complete separation may prevent possible high prices of activated distribution resources to spread in larger areas (i.e. local portions of transmission network and adjacent distribution systems). However, these high prices may be consequence of the illiquidity/scarcity caused by the separation. Therefore, CS C is expected to have low economic performance, as confirmed by simulations (Figure 10), even when sufficient reserve is allocated for distribution balancing [4]. Since this is the only scheme that implements a local balancing market, we can ascribe a share of that inefficiency also to this peculiarity, so that we can infer that balancing should be coped globally (as commonly acknowledged [24]).

\section{Common global/local ancillary services market}

CS D is characterized by a common market for both DSO and TSO that considers all technical and network constraints, but needs a considerable computational effort for its clearing. However, since all the information is shared and available to both TSO and DSO, the optimal solution can be found and we can expect high economic performances, as confirmed by simulations results. This, however, needs a strong TSO-DSO cooperation. In particular, regulation should take care of all the data sharing implications. A relevant drawback of such a scheme is the impact of forecasting error: it may cause activation of resources for solving forecasted imbalances and/or congestions that do not actually occur, so that counteractions are needed in real time. Note that forecasting errors affect all the schemes. Pushing gate closure as close as possible to activation time and/or increasing market clearing frequency could reduce this impact; however, some technical constraints may not be easily eliminated, as well as the already mentioned computational effort for the clearing of the common market with all the network constraints included.

\section{ACKNOWLEDGMENT}

SmartNet project (smartnet-project.eu) has received funding from the European Union's Horizon 2020 research and innovation program under grant agreement No 691405.

\section{REFERENCES}

[1] J. Silva, J. Sumaili, R. J. Bessa, L. Seca, M. A. Matos, V. Miranda, M. Caujolle, B. Goncer, and M. Sebastian-Viana, "Estimating the Active and Reactive Power Flexibility Area at the TSO-DSO Interface," IEEE Transactions on Power Systems, vol. 33, no. 5, 2018, pp. 4741-4750.

[2] J. Villar, R. Bessa, and M. Matos, "Flexibility products and markets: Literature review," Electric Power Systems Research, vol. 154, 2018, pp. 329-340.

[3] A. Vicente-Pastor, J. Nieto-Martin, D. W. Bunn, and A. Laur, "Evaluation of Flexibility Markets for Retailer-DSO-TSO Coordination," IEEE Transactions on Power Systems, vol. 34, no. 3, 2019, pp. 2003-2012.
[4] N. Savvopoulos, T. Konstantinou, and N. Hatziargyriou, "TSO-DSO Coordination in Decentralized Ancillary Services Markets," In Proc of 2019 International Conference on Smart Energy Systems and Technologies (SEST), 2019, pp. 1-6.

[5] ENA Open Networks Projects "Opening Markets for Network Flexibility - 2017: Achievements and Future Direction”, Report, 2017.

[6] H. H. Grøttum, S. F. Bjerland, P. C. d. Granado, and R. Egging, "Modelling TSO-DSO coordination: The value of distributed flexible resources to the power system," In Proc of 2019 16th International Conference on the European Energy Market (EEM), 2019, pp. 1-6.

[7] ENTSO E "Towards Smarter Grids: ENTSO-E Position Paper on Developing TSO and DSO Roles for the Benefit of Consumers," Position paper, March 2015.

[8] Z. Yuan, and M. R. Hesamzadeh, "Hierarchical coordination of TSODSO economic dispatch considering large-scale integration of distributed energy resources," Applied Energy, vol. 195, 2017, pp. 600615.

[9] H. Gerard, E. I. Rivero Puente, D. Six, "Coordination between transmission and distribution system operators in the electricity sector: A conceptual framework," Utilities Policy, vol. 50, pp. 40-48, 2018.

[10] H. Svendsen, M. Rossi, G. Viganò, J. Merino, J. Le Baut, H. Sawsan, "D4.2. Scenario setup and simulation results," SmartNet project deliverable, 2019.

[11] G. Leclercq, M. Pavesi, T. Gueuning, A. Ashouri, P. Sels, F. Geth, R. D'hulst, H. Le Cadre, "D2.2. Network and market models," SmartNet project deliverable, 2019.

[12] F. Pröstl Andrén, T. Strasser, J. Le Baut, M. Rossi, G. Viganò, Della G. Croce, S. Horsmanheimo, A. G. Azar, A. Ibañez, "Validating Coordination schemes between Transmission and Distribution System Operators using a Laboratory-Based Approach," IEEE PES Powertech, 2019.

[13] Kuusela P., Koponen P., Kockar I., Xu H., “An ICT Cost Comparison of Different Market Structures for Distributed Ancillary Services," in CIRED - Open Access Proceedings Journal, paper n 827, 2019.

[14] N. O'Connell, P. Pinson, H. Madsen and M. O'Malley, "Economic Dispatch of Demand Response Balancing Through Asymmetric Block Offers," in IEEE Transactions on Power Systems, vol. 31, no. 4, pp. 2999-3007, July 2016.

[15] M. Dzamarija, M. Plecas, J. Jimeno, H. Marthinsen, J. Camargo, Y. Vardanyan, M. Marroquin, D. Sánchez, F. Spiessens, G. Leclercq, N Ruiz, "D2.1. Aggregation models," SmartNet project deliverable, 2018

[16] J. Camargo, F. Spiessens, C. Hermans, "A Network Flow Model for Price-Responsive Control of Deferrable Load Profiles," Energies, vol. 11, pp. 613,2018

[17] J. Jimeno, N. Ruiz, C. Madina, "Aggregation of Thermostatically Controlled Loads for Flexibility Markets", CIRED 2019, paper $n^{\circ} 1502$, 2019.

[18] Y. Vardanyan, H. Madsen, "Optimal Coordinated Bidding of a Profit Maximizing, Risk-Averse EV Aggregator in Three-Settlement Markets Under Uncertainty," Energies, vol. 12, pp. 1755, 2019.

[19] H. Marthinsen, A. Z. Morch, M. Plećaš, I. Kockar and M. Džamarija, "Aggregation model for curtailable generation and sheddable loads," in CIRED - Open Access Proceedings Journal, vol. 2017, no. 1, pp. 15621566, 2017.

[20] M. Rossi, G. Vigano, D. Moneta and C. Carlini, "Integration of droop control functions for distributed generation in power flow simulations," 2015 AEIT International Annual Conference (AEIT), Naples, pp. 1-6, 2015.

[21] I. Gómez, S. Riaño, C. Madina, M. Rossi, P. Kuusela, P. Koponen, H. Aghaie, G. Migliavacca, E. Rivero, H. Xu, I. Kockar, "D4.3. Costbenefit analysis of the selected national cases" SmartNet project deliverable, 2019.

[22] Cedec, EDSO, ENTSOe, Eurelectric, Geode, "General Guidelines for Reinforcing the Cooperation between TSOs and DSOs," 2015

[23] S. Mathieu, D. Ernst and B. Cornéiusse, "Agent-based analysis of dynamic access ranges to the distribution network," 2016 IEEE PES Innovative Smart Grid Technologies Conference Europe (ISGTEurope), Ljubljana, 2016, pp. 1-6.

[24] Cedec, EDSO, ENTSOe, Eurelectric, Geode, “TSO-DSO Report - An Integrated Approach to Active System Management", 2019. 